\title{
Does type I interferon limit protective neutrophil responses during pulmonary Francisella tularensis infection?
}

\author{
Yoichi Furuya, Donald Steiner and Dennis W. Metzger * \\ Center for Immunology and Microbial Disease, Albany Medical College, Albany, NY, USA \\ ${ }^{*}$ Correspondence: metzged@mail.amc.edu \\ Edited by: \\ Amy Rasley, Lawrence Livermore National Laboratory, USA \\ Reviewed by: \\ David Weiss, Emory University, USA
}

Keywords: Francisella tularensis, mucosal immunity, type I interferon, immune evasion, neutrophil

\section{FRANCISELLA TULARENSIS}

Host-microorganism co-existence has enabled many pathogens to develop mechanisms to evade the immune system. One prime example is Francisella tularensis. This Gram-negative bacterium infects various wild animals such as rodents and rabbits, but also exists in water and soil. In rare cases, humans acquire $F$. tularensis infections through inhalation of particles from infected animals, drinking of contaminated water, ingestion of undercooked infected meat, or a bite from an infected tick or mosquito. Respiratory infection is the most deadly form of disease with a mortality rate as high as $50 \%$ if untreated. Due to its extreme virulence and ease of aerosol dissemination, it is classified as a Tier 1 bioterrorism agent by the Centers for Disease Control and Prevention. Given that inhalation of aerosolized $F$. tularensis would be the most likely route of transmission during an act of bioterrorism, recent research has shifted from intravenous/intradermal infection models to respiratory models of tularemia. Upon pulmonary infection, many cell types such as alveolar macrophages, neutrophils, and dendritic cells have been shown to harbor live $F$. tularensis $(1,2)$. Thus, $F$. tularensis is considered as an intracellular bacterium despite its ability to grow in a culture medium. Currently, whether phagocytes contribute to host defense or promote bacterial replication is an intense focus of biodefense research.

\section{TYPE I INTERFERON (IFN-I)-MEDIATED} SUPPRESSION OF NEUTROPHIL

\section{RECRUITMENT}

Francisella tularensis possesses a remarkable ability to evade the host innate immune response. The first line of defense against most respiratory pathogens is the alveolar macrophage, the predominant cell type found in the airways of naïve hosts. A major role for alveolar macrophages is to efficiently eliminate invading pathogens through phagocytosis. However, F. tularensis has evolved a mechanism to escape macrophage killing and to replicate within phagocytes. This, together with the relatively inert properties of the $F$. tularensis LPS, renders alveolar macrophages unable to produce the cytokines and chemokines that are necessary to initiate effective immune responses during the early phases of F. tularensis infection (3). However, macrophage infection eventually does trigger a host immune response through bacterial recognition by cytosolic receptors, which in turn, results in type I IFN (IFN-I) production $(4,5)$. IFN-I signaling stimulates expression of "absent in melanoma 2" (AIM-2), a component of the inflammasome that is required for resistance against F. tularensis infection $(5,6)$. Indeed, mice deficient in AIM-2 are highly susceptible to intradermal or subcutaneous F. tularensis challenge $(6,7)$. However, a contradictory finding is that mice deficient in IFN-I receptors (IFN-IR ${ }^{-1}$ ) exhibit substantially increased resistance to intranasal and intradermal Francisella infection (8, 9). This resistance suggests that the detrimental effects of IFN-I outweigh the benefits of IFN-I-mediated AIM-2 expression that is required for the inflammasome response. The detrimental effects of IFNI were ascribed to its negative influence on expression of $\gamma \delta \mathrm{T}$ cells that produce IL-17 (8). Increased survival of IFN-IR ${ }^{-/-}$ mice following subcutaneous tularemia was closely associated with an increased
IL-17 response, reduced bacterial burden, and increased influx of neutrophils into the spleen (8). Suppression of IL-17 responses and neutrophil recruitment by IFN-I has similarly been reported in other infectious disease models (10). These various findings have led to the hypothesis that IL-17mediated neutrophil recruitment is protective against $F$. tularensis infection. Consistent with this report, IL-17A deficient mice were found to be more susceptible to intranasal F. tularensis infection (11-13). However, although a role for IL-17 in facilitating neutrophil responses is well established (14), during pneumonic tularemia, recruitment of $\mathrm{CD} 11 \mathrm{~b}^{+} \mathrm{Ly} 6 \mathrm{G} / \mathrm{C}^{+}$cells was not affected by the absence of IL-17A (13). This suggests that IFN-I may limit neutrophil infiltration during pulmonary $F$. tularensis infection independently from its effects on IL-17 expression. It would be of considerable interest to assess production of neutrophil chemoattractants, such as CXCL1 and CXCL2 in the lungs of IFN$\mathrm{IR}^{-1-}$ mice following intranasal F. tularensis infection, to elucidate the mechanisms responsible for IFN-I-mediated suppression of neutrophil recruitment.

An alternative or complementary mechanism that may be responsible for decreased neutrophil recruitment to the site of infection by IFN-I involves host cell death. Immune cells such as lymphocytes, macrophages, and neutrophils can be sensitized by IFN-I toward cell death in a mouse model of Francisella and Listeria monocytogenes infection (5, 15-17). Consistent with the findings in mice, human neutrophils with upregulated interferon-stimulated gene expression exhibit enhanced cell death following in vitro exposure to Staphylococcus aureus 
(15). This phenomenon, however, seems to be pathogen-specific since enhanced cell death was not observed after exposure to Pseudomonas aeruginosa (15). Indeed, spontaneous death of neutrophils in the absence of pathogen can be delayed by in vitro IFN-I treatment (1). Thus, the direct effect of IFN-I signaling on neutrophils could be context-dependent. In the case of Francisella infection, IFN-I signaling may promote neutrophil death and contribute to the overall decrease in neutrophil numbers at the site of infection.

\section{CONTROVERSIAL ROLE OF NEUTROPHILS DURING PULMONARY TULAREMIIA}

Neutrophils make up the first wave of phagocytic cell migration into the lungs during most pulmonary bacterial infections, including respiratory tularemia (2). However, previous studies utilizing the neutrophil depleting antibody, anti-Gr1, have failed to show an important role for neutrophils during pulmonary F. tularensis infection, as assessed by bacterial burden (18). This is in striking contrast to the known importance of neutrophils in host defense against systemic tularemia (18-20). Recently, it has been proposed that neutrophils may even promote immunopathology during pulmonary tularemia. Mice deficient in metalloproteinase-9 (MMP-9), a mediator of leukocyte migration, have reduced neutrophil recruitment into the lungs following intranasal $F$. tularensis infection (21). Surprisingly, these mice exhibit better survival compared to wild-type mice following pulmonary tularemia. The interpretation from this study was that the excessive neutrophil infiltration observed in wild-type mice was detrimental to the host, but the limited recruitment of neutrophils seen in $\mathrm{MMP}-9^{-1-}$ mice was beneficial (21). We have recently found that near complete depletion of neutrophils using a neutrophil-specific mAb, anti-Ly6G, significantly reduced survival of intranasal Francisella-infected mice, an observation that is consistent with a protective role for low to moderate recruitment of neutrophils (unpublished observations). Overall, the accumulating evidence suggests that neutrophils do play a role during pneumonic tularemia, but it remains uncertain whether neutrophils exert protective or harmful effects during pneumonic tularemia. It is important to note that some of the discrepancies in results may be due to the differences in experimental conditions such as the type of in vivo depleting antibody, timing of antibody administration, and/or mouse strains.

\section{IFN-IR ${ }^{-/-}$MICE AS A MODEL TO STUDY PROTECTIVE ROLE OF NEUTROPHILS DURING PULMONARY TULAREMIA}

We propose that IFN-IR ${ }^{-/}$mice can serve as a valuable tool to better understand the role of IFN-I and neutrophils in defense of mucosal tissues against pulmonary tularemia. To the best of our knowledge, only a few studies have been performed using IFN-IR ${ }^{-1-}$ mice in the pulmonary tularemia model. It would be of considerable interest to determine whether the absence of IFN-I signaling alters the kinetics of pulmonary neutrophil recruitment during respiratory tularemia. If so, an important question to address is whether in vivo depletion of neutrophils during the early versus late phases of pulmonary tularemia in $\mathrm{IFN}^{-\mathrm{IR}^{-1}}$ mice results in a differential survival outcome. Perhaps in the absence of IFN-I signaling, there is an early influx of neutrophils into the lungs, which promotes early bacterial clearance, but later recruitment is not affected or reduced. This scenario would be consistent with the concept that neutrophils are detrimental if pulmonary infiltration is excessive (21), but their complete absence results in reduced survival. A fine balance may exist between the protective role of neutrophils during the early phase of bacterial infection versus excessive neutrophil recruitment during the recovery phase, which impedes resolution of pulmonary inflammation (depicted in Figure 1). An

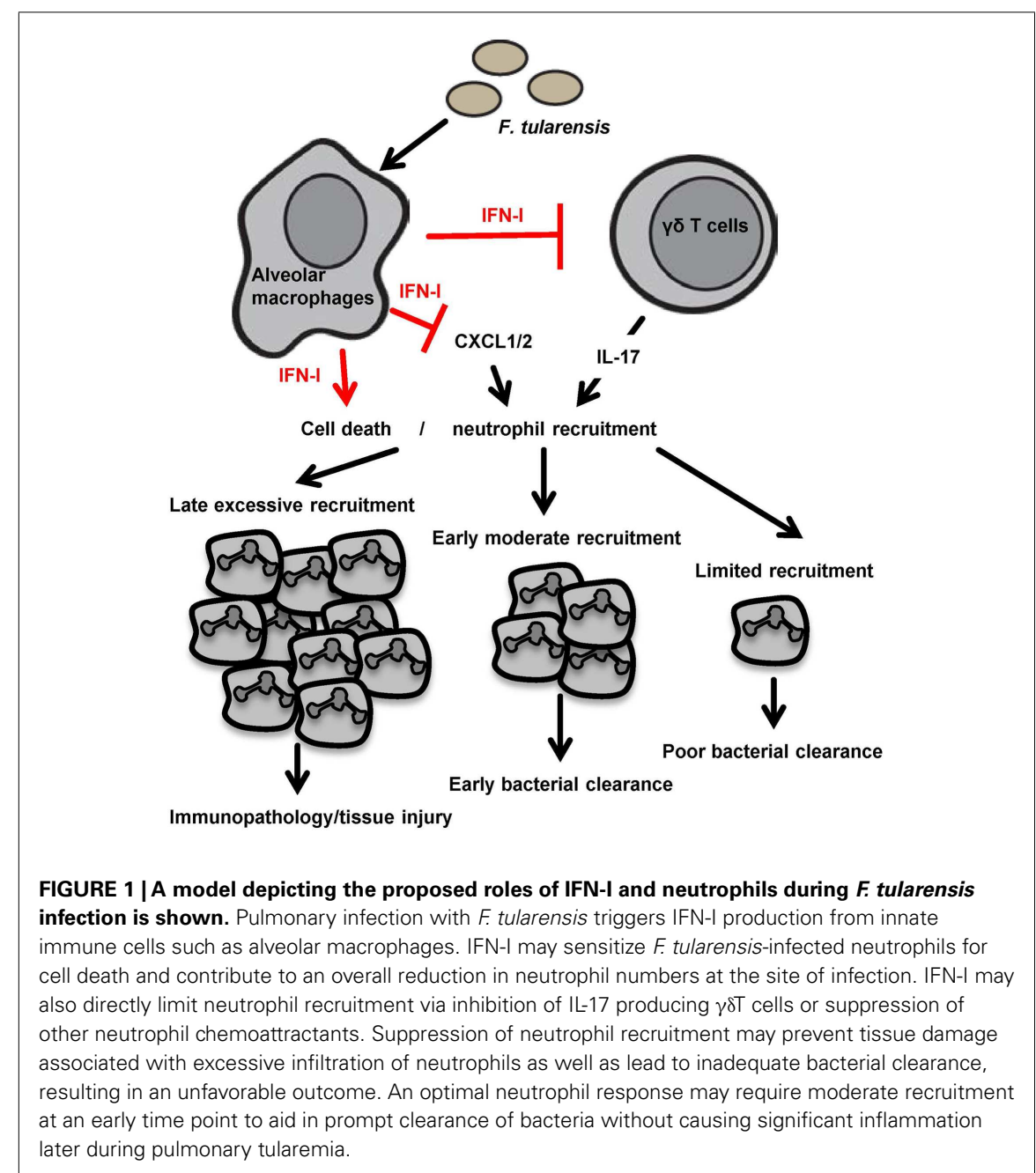


increased understanding of neutrophilmediated mucosal immunity and the role IFN-I signaling in regulating recruitment of these cells to the lung may ultimately facilitate the development of novel therapeutics for protection against tularemia.

\section{ACKNOWLEDGMENTS}

This work was supported by an American Lung Association Senior Research Training Fellowship RT-226959-N to Yoichi Furuya and by NIH grant PO1 AI056320.

\section{REFERENCES}

1. Allen LA. Interview with Dr. Lee-Ann Allen regarding pivotal advance: Francisella tularensis LVS evades killing by human neutrophils via inhibition of the respiratory burst and phagosome escape. Interview by Helene F. Rosenberg. J Leukoc Biol (2006) 80(6):1222-3. doi:10.1189/jlb.1306287

2. Hall JD, Woolard MD, Gunn BM, Craven RR, TaftBenz S, Frelinger JA, et al. Infected-host-cell repertoire and cellular response in the lung following inhalation of Francisella tularensis Schu S4, LVS, or U112. Infect Immun (2008) 76(12):5843-52. doi:10.1128/IAI.01176-08

3. Singh A, Rahman T, Malik M, Hickey AJ, Leifer CA, Hazlett KR, et al. Discordant results obtained with Francisella tularensis during in vitro and in vivo immunological studies are attributable to compromised bacterial structural integrity. PLoS One (2013) 8(3):e58513. doi:10.1371/journal. pone. 0058513

4. Stetson DB, Medzhitov R. Recognition of cytosolic DNA activates an IRF3-dependent innate immune response. Immunity (2006) 24(1):93-103. doi:10. 1016/j.immuni.2005.12.003

5. Henry T, Brotcke A, Weiss DS, Thompson LJ, Monack DM. Type I interferon signaling is required for activation of the inflammasome during Francisella infection. J Exp Med (2007) 204(5):987-94. doi:10.1084/jem.20062665

6. Jones JW, Kayagaki N, Broz P, Henry T, Newton $\mathrm{K}$, O'Rourke $\mathrm{K}$, et al. Absent in melanoma 2 is required for innate immune recognition of Francisella tularensis. Proc Natl Acad Sci U S A (2010) 107(21):9771-6. doi:10.1073/pnas.1003738107

7. Fernandes-Alnemri T, Yu JW, Juliana C, Solorzano L, Kang S, Wu J, et al. The AIM2 inflammasome is critical for innate immunity to Francisella tularensis. Nat Immunol (2010) 11(5):385-93. doi:10. 1038/ni.1859

8. Henry T, Kirimanjeswara GS, Ruby T, Jones JW, Peng K, Perret $\mathrm{M}$, et al. Type I IFN signaling constrains IL-17A/F secretion by gammadelta T cells during bacterial infections. J Immunol (2010) 184(7):3755-67. doi:10.4049/jimmunol.0902065

9. Metzger DW, Bakshi CS, Kirimanjeswara G. Mucosal immunopathogenesis of Francisella tularensis. Ann N Y Acad Sci (2007) 1105:266-83. doi:10.1196/annals.1409.007

10. Li W, Moltedo B, Moran TM. Type I interferon induction during influenza virus infection increases susceptibility to secondary Streptococcus pneumoniae infection by negative regulation of gammadelta T cells. J Virol (2012) 86(22):12304-12. doi:10.1128/JVI.01269-12

11. Skyberg JA, Rollins MF, Samuel JW, Sutherland MD, Belisle JT, Pascual DW. Interleukin-17 protects against the Francisella tularensis live vaccine strain but not against a virulent $F$. tularensis type A strain. Infect Immun (2013) 81(9):3099-105. doi:10.1128/IAI.00203-13

12. Markel G, Bar-Haim E, Zahavy E, Cohen H, Cohen O, Shafferman A, et al. The involvement of IL-17A in the murine response to sub-lethal inhalational infection with Francisella tularensis. PLoS One (2010) 5(6):e11176. doi:10.1371/ journal.pone.0011176

13. Cowley SC, Meierovics AI, Frelinger JA, Iwakura Y, Elkins KL. Lung CD4-CD8- double-negative T cells are prominent producers of IL-17A and IFNgamma during primary respiratory murine infection with Francisella tularensis live vaccine strain. J Immunol (2010) 184(10):5791-801. doi:10.4049/ jimmunol.1000362

14. Iwakura $Y$, Nakae S, Saijo S, Ishigame $H$. The roles of IL-17A in inflammatory immune responses and host defense against pathogens. Immunol Rev (2008) 226:57-79. doi:10.1111/j.1600-065X.2008. 00699.x

15. Malcolm KC, Kret JE, Young RL, Poch KR, Caceres SM, Douglas IS, et al. Bacteria-specific neutrophil dysfunction associated with interferonstimulated gene expression in the acute respiratory distress syndrome. PLoS One (2011) 6(7):e21958. doi:10.1371/journal.pone.0021958

16. Carrero JA, Calderon B, Unanue ER. Lymphocytes are detrimental during the early innate immune response against Listeria monocytogenes. J Exp Med (2006) 203(4):933-40. doi:10.1084/jem. 20060045
17. Stockinger S, Materna T, Stoiber D, Bayr L, Steinborn R, Kolbe T, et al. Production of type I IFN sensitizes macrophages to cell death induced by Listeria monocytogenes. I Immunol (2002) 169(11):6522-9. doi:10.4049/jimmunol. 169.11.6522

18. Conlan JW, KuoLee R, Shen H, Webb A. Different host defences are required to protect mice from primary systemic vs pulmonary infection with the facultative intracellular bacterial pathogen, Francisella tularensis LVS. Microb Pathog (2002) 32(3):127-34. doi:10.1006/mpat.2001.0489

19. Sjostedt A, Conlan JW, North RJ. Neutrophils are critical for host defense against primary infection with the facultative intracellular bacterium Francisella tularensis in mice and participate in defense against reinfection. Infect Immun (1994) 62(7):2779-83.

20. Elkins KL, Rhinehart-Jones TR, Culkin SJ, Yee D, Winegar RK. Minimal requirements for murine resistance to infection with Francisella tularensis LVS. Infect Immun (1996) 64(8):3288-93.

21. Malik M, Bakshi CS, McCabe K, Catlett SV, Shah A, Singh R, et al. Matrix metalloproteinase 9 activity enhances host susceptibility to pulmonary infection with type A and B strains of Francisella tularensis. J Immunol (2007) 178(2):1013-20. doi: 10.4049/jimmunol.178.2.1013

Conflict of Interest Statement: The authors declare that the research was conducted in the absence of any commercial or financial relationships that could be construed as a potential conflict of interest.

Received: 16 May 2014; paper pending published:07 June 2014; accepted: 09 July 2014; published online: 23 July 2014.

Citation: Furuya $Y$, Steiner $D$ and Metzger DW (2014) Does type I interferon limit protective neutrophil responses during pulmonary Francisella tularensis infection? Front. Immunol. 5:355. doi: 10.3389/fimmu.2014.00355

This article was submitted to Microbial Immunology, a section of the journal Frontiers in Immunology.

Copyright (c) 2014 Furuya, Steiner and Metzger. This is an open-access article distributed under the terms of the Creative Commons Attribution License (CC BY). The use, distribution or reproduction in other forums is permitted, provided the original author(s) or licensor are credited and that the original publication in this journal is cited, in accordance with accepted academic practice. No use, distribution or reproduction is permitted which does not comply with these terms. 